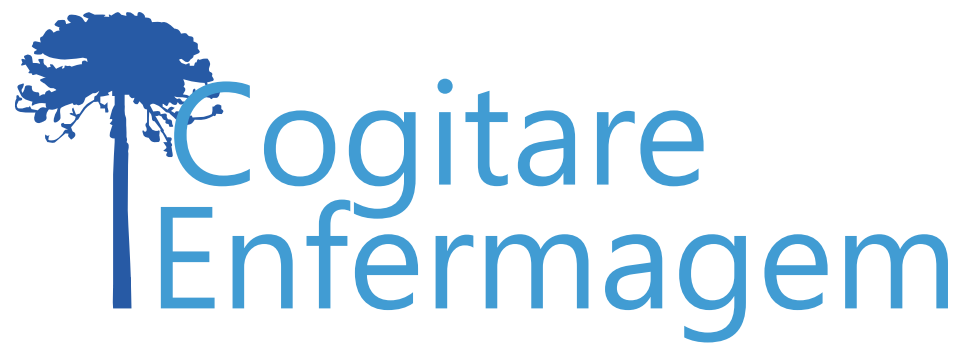

\title{
EFEITOS DA MAMOPLASTIA REDUTORA NA FUNÇÃO PULMONAR E QUALIDADE DE VIDA DE MULHERES SUBMETIDAS À GIGANTOPLASTIA
}

Olympio José dos Santos ${ }^{1}$, Carlos Lyra da Silva², Thiago Quinellato Louro ${ }^{3}$, Daniel Aragão Machado ${ }^{4}$ João Guilherme Novis de Souza Avellar ${ }^{5}$, Roberto Carlos Lyra da Silva ${ }^{6}$

\section{RESUMO}

Objetivo: avaliar os efeitos da mamoplastia redutora na função pulmonar e qualidade de vida de mulheres com gigantomastia.

Método: estudo observacional do tipo série de casos que analisou retrospectivamente o prontuário de 14 casos de mulheres submetidas à mamoplastia redutora nos últimos 12 meses no serviço de clínica cirúrgica de um hospital universitário do Rio de Janeiro.

Resultados: a média de idade, altura e peso das mulheres submetidas à mamoplastia foi respectivamente, 44,5 anos, 1,58 m e 93,8 kg. O índice de massa corporal evidenciou obesidade de graus I a III. Em média a espirometria variou de $92,76 \%$ do valor teórico predito antes da cirurgia, para 94,74\% e 91,77\%, 30 e 60 dias após a cirurgia respectivamente.

Conclusão: a mamoplastia de redução pode melhorar a qualidade de vida das mulheres, conforme autorrelato. Não houve associação da mamoplastia com a melhora da função respiratória.

DESCRITORES: Mamoplastia; Qualidade de Vida; Espirometria; Cirurgia Plástica; Enfermagem Cirúrgica.

\section{COMO REFERENCIAR ESTE ARTIGO:}

Santos OJ dos, Silva CL da, Louro TQ, Machado DA, Avellar JGN de S, Silva RCL da. Efeitos da mamoplastia redutora na função pulmonar e qualidade de vida de mulheres submetidas à gigantoplastia. Cogitare enferm. [Internet]. 2019 [acesso em "colocar data de acesso, dia, mês abreviado e ano"]; 24. Disponível em: http:// dx.doi.org/10.5380/ce.v24i0.64034.

Este obra está licenciado com uma Licença Creative Commons Atribuição 4.0 Internacional.

${ }^{1}$ Médico. Pós-Doutor em Enfermagem e Biociências, Doutor em Cirurgia Plástica. Docente da Universidade Federal Fluminense. Rio de Janeiro, RJ, Brasil.

${ }^{2}$ Enfermeiro. Pós-Doutor em Enfermagem. Docente de Pós-Graduação em Enfermagem e Biociências da Universidade Federal do Rio de Janeiro. Rio de Janeiro, RJ, Brasil. 9

${ }^{3}$ Enfermeiro. Pós-Doutorando em Enfermagem e Biociências. Docente de Enfermagem da Universidade Federal Fluminense. Rio das Ostras, RJ, Brasil. (2)

${ }^{4}$ Enfermeiro. Doutor em Enfermagem e Biociências. Docente de Pós-Graduação em Tecnologias no Espaço Hospitalar da Universidade Federal do Estado do Rio de Janeiro. Rio de Janeiro, RJ, Brasil. 9

${ }^{5}$ Discente de Medicina. Universidade Federal Fluminense. Rio de Janeiro, RJ, Brasil.

${ }^{6}$ Enfermeiro. Doutor em Enfermagem. Docente de Pós-Graduação em Enfermagem e Biociências da Universidade Federal do Rio de Janeiro. Rio de Janeiro, RJ, Brasil. 


\title{
EFFECTS OF REDUCTION MAMMOPLASTY ON THE PULMONARY FUNCTION AND THE QUALITY OF LIFE OF WOMEN WITH GIGANTOMASTIA
}

\begin{abstract}
Objective: To evaluate the effects of reduction mammoplasty on the pulmonary function and quality of life of women with gigantomastia.

Method: Observational study (a case series) that retrospectively analyzed the medical records of 14 cases of women who underwent reduction mammoplasty in the last 12 months at the surgical clinic service of a university hospital in Rio de Janeiro.

Results: The mean age, height and weight of the women who underwent mammoplasty were, respectively, 44.5 years, $158 \mathrm{~cm}$ and $93.8 \mathrm{~kg}$. Body mass index revealed obesity classes ranging from I to III. Regarding spirometry, the values of FVC ranged from $92.76 \%$ of the predicted theoretical value before surgery to $94.74 \%$ and $91.77 \%$, respectively 30 and 60 days after surgery.

Conclusion: Reduction mammoplasty can improve women's quality of life, according to the reports of the participants. There was no association between mammoplasty and improvement of the respiratory function.
\end{abstract}

DESCRIPTORS: Mammoplasty; Quality of Life; Spirometry; Plastic Surgery; Surgical Nursing.

\section{EFECTOS DE MAMOPLASTÍA REDUCTORA EN FUNCIÓN PULMONAR; CALIDAD DE VIDA DE MUJERES SOMETIDAS A GIGANTOPLASTIIA}

\begin{abstract}
RESUMEN
Objetivo: Evaluar efectos de la mamoplastía reductora en función pulmonar y calidad de vida de mujeres con gigantomastía.

Método: Estudio observacional tipo serie de casos, con análisis retrospectivo de historias clínicas de 14 casos de mujeres sometidas a mamoplastía reductora en los últimos 12 meses en servicio de cirugía clínica de hospital universitario de Rio de Janeiro.

Resultados: La media etaria, altura y peso de las mujeres sometidas a mamoplastía fue, respectivamente: 44,5 años, 1,58 m y 93,8 kg. El índice de masa corporal evidenció obesidad de grados I a III. En promedio, la espirometría varió del 93,76\% del valor teórico previsto antes de la cirugía al 94,74\% a los 30 días de la cirugía y 91,77\% a los 60.

Conclusión: La mamoplastía reductora puede mejorar la calidad de vida de las mujeres, según su testimonio. No existió asociación de la mamoplastía con mejoras en la función respiratoria.
\end{abstract}

DESCRIPTORES: Mamoplastía; Calidad de Vida; Espirometría; Cirugía Plástica; Enfermería Quirúrgica. 
A gigantomastia é uma condição rara caracterizada pelo crescimento excessivo do tecido mamário que pode ser fisicamente e psicossocialmente incapacitante para a paciente. Muitos autores consideram gigantomastia o aumento de mama que requer redução de mais de $1500 \mathrm{~g}$ por mama. Contudo, há discordância na literatura com o peso da redução, variando de 0,8 a $2 \mathrm{~kg}$. Embora não exista ainda uma definição universalmente aceita, em muitas referências a definição de gigantomastia está diretamente associada ao excesso de peso do tecido mamário. Parece haver maior consenso em relação ao tratamento cirúrgico, apontado como o pilar do tratamento para Gigantomastia, na forma de mamoplastia de redução(1).

Não se conhece a incidência e prevalência da gigantomastia e muito menos a morbimortalidade. Estima-se que as complicações mais comumente incluem mastalgia, ulceração da mama e infecção, problemas posturais, dor nas costas e lesão traumática crônica aos nervos intercostais $4^{\circ} / 5^{\circ} / 6^{\circ}$, com perda resultante da sensação do mamilo e diminuição do crescimento fetal durante a gravidez $z^{(1-2)}$, complicações que podem diminuir significativamente a qualidade de vida de mulheres acometidas.

As principais queixas das mulheres com gigantomastia, e que levam à indicação da mamoplastia redutora, incluem desde a melhoria da forma das mamas a sintomas somáticos. Embora existam evidências de que esta cirurgia melhore significantemente esses sintomas, com melhora da qualidade de vida das pacientes, ainda assim, em muitos casos, a realização deste procedimento tem sido considerada uma intervenção muito mais estética do que terapêutica ${ }^{(2-3)}$.

Estudos revelam o quanto a gigantomastia concorre para alterar o componente funcional, com transtornos de ordem circulatória, respiratória e postural. Entre os transtornos respiratórios, a disfunção pulmonar é uma complicação associada à gigantomastia, ainda pouco estudada e documentada na literatura, cujas alterações estão relacionadas à função pulmonar, afetando suas capacidades e volumes, que podem ser avaliadas por meio dos testes de função pulmonar: espirometria e medida dos gases sanguíneos arteriais ${ }^{(4-6)}$.

As mamas volumosas e pesadas das pacientes, a maioria com obesidade associada, além de problemas estéticos, podem causar significativa restrição e redução da complacência da parede torácica, interferência na ventilação pulmonar, e consequentemente diminuição na qualidade de vida.

Por essa razão, a disfunção pulmonar e o comprometimento da capacidade funcional, interpretado como a capacidade de a paciente realizar atividades diárias comuns e um dos parâmetros de avaliação de qualidade de vida, precisam ser consideradas na avaliação de enfermeiros, médicos e fisioterapeutas como importantes indicadores e parâmetros fisiológicos potenciais para as complicações e limitações no corpo de mulheres acometidas por gigantomastia. Não obstante, suas condutas compatíveis com a minimização dos efeitos deletérios, a começar pela indicação e realização da mamoplastia redutora, que confira a esta intervenção cirúrgica um caráter muito mais terapêutico do que estético.

Com base no exposto, a questão estruturada de pesquisa foi: quais os efeitos da mamoplastia redutora sobre espirometria e sobre a qualidade de vida de mulheres portadoras de gigantomastia?

Para responder a esta questão, objetivou-se avaliar os efeitos da cirurgia de mamoplastia redutora sobre a função pulmonar e qualidade de vida de mulheres com gigantomastia, assim como: estimar a Capacidade Vital Forçada (CVF), a Ventilação Voluntária Máxima (VVM), o Volume Expiratório Forçado no primeiro segundo (VEF), o Fluxo Expiratório Forçado (FEF), o Índice de Tiffeneau (VEF /CVF), FEF25-75/CVF e a qualidade de vida de mulheres com gigantomastia, antes e depois de terem sido submetidas à mamoplastia redutora. 
Estudo observacional do tipo série de casos, no qual foram analisados, retrospectivamente, todos os casos de pacientes submetidas à cirurgia de mamoplastia redutora nos últimos 12 meses, no serviço de clínica cirúrgica de um hospital universitário.

A amostra foi constituída por conveniência e incluiu 14 pacientes do sexo feminino, portadoras de gigantomastia, submetidas à mamoplastia de redução. O tempo de seguimento foi de seis meses após a alta hospitalar, e os desfechos avaliados em intervalos de 30 e 60 dias após a realização do procedimento cirúrgico. Os dados de interesse para análise dos desfechos foram extraídos dos prontuários dos pacientes. Cabe destacar que, segundo consta nos prontuários, todos os exames de espirometria foram realizados em uma clínica particular utilizando o espirômetro Koko® SX 1000, tipo PC-BASED SPIROMETRY, e foram executados pelo mesmo profissional em todos os casos analisados.

Foram critérios de elegibilidade: mulheres com idades entre 18 e no máximo 60 anos e Índice de Massa Corpórea (IMC) igual ou superior a $23 \mathrm{~kg} / \mathrm{m}^{2}$, tabagistas ou não, com ou sem antecedentes médicos, e que concordaram em participar do estudo.

Todas as pacientes foram submetidas à técnica cirúrgica de Costa Lima, associada ao retalho de pedículo vascular inferior, sob anestesia geral. Optou-se por essa técnica por ser a mais frequentemente utilizada para a redução mamária de pacientes com gigantomastia ${ }^{(3)}$.

Os dados foram analisados estatisticamente pelo programa R. A análise estatística descritiva foi utilizada para calcular a distribuição das variáveis, a partir da média e do desvio padrão. As diferenças entre as médias foram medidas para estimar a diferença na magnitude do efeito da cirurgia sobre as variáveis da espirometria (VVM, CVF, VEF/CVF e FEF 25\%-75\%/CVF), para avaliar comprometimentos restritivos antes e após a realização da cirurgia.

A qualidade de vida foi avaliada de forma qualitativa, a partir de um questionário elaborado pelos próprios pesquisadores, contendo quatro perguntas abertas, a saber: 1O que mudou em sua vida após a cirurgia? 2- Como se encontra o seu estado físico depois da cirurgia? 3- Qual o seu nível de satisfação após a cirurgia? 4- O resultado da cirurgia melhorou sua autoestima?

A pesquisa teve seu protocolo aprovado pelo Comitê de Ética em Pesquisa da UNIRIO, com parecer $n^{\circ}$ 2.719.674.

\section{RESULTADOS}

A Tabela 1 nos permite conhecer a distribuição da faixa etária das mulheres que participaram do estudo (média de idade de $44,5( \pm 8,12$ anos)) e as características antropométricas dessas mulheres na linha de base.

Tabela 1 - Perfil das participantes na linha de base. Rio de Janeiro, RJ, Brasil, 2018 (continua)

\begin{tabular}{ccccc} 
Paciente & Idade & Altura $\mathbf{m}$ & Peso kg & IMC \\
\hline 1 & 37 & 1,61 & 83 & 32,02 \\
\hline 2 & 35 & 1,63 & 99 & 37,26 \\
\hline 3 & 42 & 1,68 & 85 & 30,11 \\
\hline
\end{tabular}




\begin{tabular}{ccccc}
\hline 4 & 61 & 1,52 & 71 & 30,73 \\
\hline 5 & 52 & 1,65 & 84 & 30,85 \\
\hline 6 & 34 & 1,62 & 97 & 36,96 \\
\hline 7 & 38 & 1,59 & 100 & 39,55 \\
\hline 8 & 52 & 1,66 & 110 & 39,91 \\
\hline 9 & 38 & 1,44 & 77 & 37,13 \\
\hline 10 & 43 & 1,55 & 113 & 47,03 \\
\hline 11 & 45 & 1,52 & 108 & 46,74 \\
\hline 12 & 44 & 1,5 & 90 & 40 \\
\hline 13 & 48 & 1,56 & 103 & 42,32 \\
\hline 14 & 55 & 1,62 & 94 & 35,81 \\
\hline Média & 44,57 & 1,58 & 93,85 & 37,6 \\
\hline DP & 8,12 & 0,06 & 12,7 & 5,5
\end{tabular}

A média da altura foi de $158,2 \mathrm{~cm}$ ( $\pm 6,93$ centímetros). Em relação ao peso, a média foi de 93,8 $( \pm 12,70 \mathrm{~kg})$, com mediana de $95 \mathrm{~kg}$.

O IMC apresentou uma média de $37,60( \pm 5,50)$, revelando que as participantes do estudo estão classificadas em obesas graus I, quatro mulheres $(33,33 \%)$ IMC de 30 a 34,9 , grau II, seis mulheres (50\%) IMC de 35 a 39,9 e grau III ou obesidade mórbida, duas mulheres $(16,33 \%)$ com IMC maior que 40.

Com relação à espirometria, foi possível constatar que a CVF (Tabela 2) variou em média entre $92,76 \%$ do valor teórico predito (média de 3,12 $\pm 0,344 \mathrm{~L} / \mathrm{min}$ ), antes da cirurgia, e $94,74 \%$ e $91,77 \%$, após a cirurgia (entre 30 e 60 dias após), demonstrando que a cirurgia não impactou significativamente essa variável.

Tabela 2 - Distribuição da referência teórica e das estimativas da variável espirométrica CFV. Rio de Janeiro, RJ, Brasil, 2018 (continua)

\begin{tabular}{ccccc} 
Paciente & Teórica & Pré-Cir. & 1 $^{\text {a }}$ Pós-Cir. & 2 $^{\text {a }}$ Pós-Cir. \\
\hline 1 & 3,55 & 3,24 & 3,28 & 3,36 \\
\hline 2 & 3,45 & 3,2 & 3,7 & 3,26 \\
\hline 3 & 3,55 & 3,45 & 3,41 & 3,35 \\
\hline 4 & 2,52 & 2,4 & 2,52 & 2,63 \\
\hline 5 & 3,45 & 2,65 & 2,64 & 2,7 \\
\hline 6 & 3,42 & 3,83 & 3,76 & 3,69 \\
\hline 7 & 3,22 & 3,05 & 2,94 & 2,99 \\
\hline 8 & 3,3 & 2,7 & 3,02 & 2,87 \\
\hline 9 & 2,62 & 3,11 & 3,18 & 3,09 \\
\hline 10 & 2,96 & 3,2 & 3,32 & 3,19 \\
\hline
\end{tabular}




\begin{tabular}{ccccc}
\hline 11 & 2,79 & 2,78 & 2,64 & 2,42 \\
\hline 12 & 2,94 & 2,15 & 2,11 & 2,34 \\
\hline 13 & 2,92 & 2,44 & 2,42 & 1,99 \\
\hline 14 & 3,07 & 2,39 & 2,26 & 2,28 \\
\hline Média & 3,12 & 2,89 & 2,94 & 2,86 \\
\hline DP & 0,34 & 0,47 & 0,52 & 0,49
\end{tabular}

A CVF representa o volume máximo de ar exalado com esforço máximo, o que se dá a partir do ponto de máxima inspiração. O resultado da CVF costuma ser expresso em valores absolutos e em percentual de predito. Quando estiver abaixo de $80 \%$ do previsto, na presença de VEF1/CVF normal, sugere distúrbio restritivo.

Não foram encontrados indícios de distúrbios restritivos nos casos estudados nem antes e nem após a realização da cirurgia, durante o tempo de seguimento. A realização da cirurgia não modificou a média da CFV nem na primeira avaliação pós-cirurgia e nem na segunda $(2,94$ e $2,96 \mathrm{~L} / \mathrm{min}$ respectivamente). Todas as duas medidas permaneceram discretamente abaixo da média esperada que era de 3,12 L/min.

CVF é o teste de função pulmonar mais importante porque num dado indivíduo, durante a expiração, existe um limite para o fluxo máximo que pode ser atingido em qualquer volume pulmonar.

Com relação à $V V M$, podemos verificar na Tabela 3 que, em média, houve um discreto incremento da VVM após 60 dias da cirurgia, na ordem de 1,65 L/min. embora ainda estivesse bastante distante do valor predito em média (101,85 L/min.). Entre as mulheres com obesidade mórbida (pacientes 10, 11 e 13), não foi verificada melhora significativa da VVM.

Tabela 3 - Distribuição da referência teórica e das estimativas da variável espirométrica WVM. Rio de Janeiro, RJ, Brasil, 2018 (continua)

\begin{tabular}{ccccc} 
Paciente & Teórica & Pré-Cir. & 1 $^{\text {a }}$ Pós-Cir. & 2 $^{\text {a }}$ Pós-Cir. \\
\hline 1 & 119,28 & 105,89 & 98,16 & 101,34 \\
\hline 2 & 108,25 & 90,58 & 96,37 & 102,27 \\
\hline 3 & 109,47 & 110,59 & 108,82 & 105,52 \\
\hline 4 & 76,46 & 72,78 & 65,05 & 83,44 \\
\hline 5 & 107,88 & 77 & 77,07 & 76,18 \\
\hline 6 & 107,72 & 123,28 & 121,69 & 120,51 \\
\hline 7 & 101,22 & 83,25 & 87,02 & 89,95 \\
\hline 8 & 99,98 & 78,88 & 79,02 & 83,73 \\
\hline 9 & 84,73 & 93,06 & 94,5 & 91,69 \\
\hline 10 & 92,8 & 96,27 & 96,99 & 98,24 \\
\hline 11 & 87,7 & 86,8 & 81,44 & 84,88 \\
\hline 12 & 113,96 & 53,06 & 62,38 & 66,75 \\
\hline 13 & 110,92 & 66,46 & 64,79 & 57,76 \\
\hline
\end{tabular}




\begin{tabular}{ccccc}
\hline 14 & 105,6 & 71,26 & 69,75 & 69,97 \\
\hline Média & 101,85 & 86,36 & 85,93 & 88,01 \\
\hline DP & 12,21 & 18,73 & 17,76 & 16,97
\end{tabular}

A VVM representa o volume máximo de ar ventilado em um período, por repetidas manobras respiratórias forçadas. O teste dá uma visão global inespecífica da função ventilatória. As unidades usadas são L/min. Considerando o valor esperado de VVM médio de acordo as características das participantes do estudo (VVM=101,85 L/min), foi possível constatar que somente na segunda avaliação espirométrica, com até 60 dias após a cirurgia, houve melhora na VVM estimada, comparado ao momento anterior a realização da cirurgia $(86,36 \times 88,01 \mathrm{~L} / \mathrm{min})$, apresentando um incremento médio de 1,64 L/min.

$\mathrm{Na}$ espirometria realizada em até 30 dias após a cirurgia, o VVM médio foi estimado em 85,93 L/min, uma redução na VVM de 0,43 L/min, o que pode ser explicado, entre outras coisas, pelas próprias limitações de expansibilidade torácica, imposta pela dor, considerando o intervalo de tempo entre a realização da cirurgia e a primeira espirometria. A cirurgia foi capaz de melhorar a WVM somente entre as mulheres com idades entre $30 \mathrm{e}$ 45 anos, passando de 93,64 L/min para 94,15 L/min em até 30 dias após a cirurgia e para $95,68 \mathrm{~L} / \mathrm{min}$ em até 60 dias depois. As mulheres obesas grau II apresentaram uma melhora média de 90,05 L/min para 93,02 L/min, em até 60 dias após a cirurgia, um incremento de $2,97 \mathrm{~L} / \mathrm{min}$.

A VVM testa a capacidade do indivíduo para sustentar um alto nível de ventilação. Valores anormais da VVM são demonstrados quando um indivíduo tem doença restritiva clinicamente significante, ou especialmente obstrutiva. Nesse sentido, o fato de ter sido observado um pequeno incremento na VVM após a realização da cirurgia, mesmo que em até 60 dias depois, mostra o quanto a gigantoplastia pode melhorar a capacidade ventilatória dessas pacientes, uma vez que o sucesso na medida de um valor representativo da VVM depende grandemente do esforço do paciente.

Foram calculados o Odds Ratio (OR) para estimar a chance de melhora do VVM, fazendo comparações entre o grupo de mulheres com obesidade grau I e grau II; entre o grupo de mulheres com obesidade grau I e grau III e entre o grupo de mulheres com obesidade grau II e grau III e considerando os valores da VVM antes da cirurgia e 60 dias depois.

O OR na primeira comparação foi de 0,33 (Intervalo de Confiança (IC) 95\%: 0,0208 a $5,3294) p=0,43$, na segunda, de 0,33 (IC $95 \%$ : 0,01 a 6,65) e na última, de 1,0 (IC $95 \% \mathrm{Cl}$ : $0,07$ a 12,55$) p=1,0$. Sem significância estatística em todas as comparações.

Comparações foram feitas também com relação à faixa etária e o VVM. Foram comparadas mulheres entre 35 e 45 anos e mulheres com mais de 45 anos em relação à chance de apresentar melhora na VVM. Mulheres com menos de 45 anos apresentaram 1,2 vezes mais chances de melhorar a VVM após a cirurgia do que aquelas com mais de 45 anos (OR 1,2 IC 95\%: 0,1303 a 11,0529) $p=0,8721$, sem significância estatística.

Cabe destacar que é possível para o indivíduo com doença pulmonar restritiva ter valores para WVM dentro da faixa normal. Isto ocorre porque eles são capazes de compensar a falta de aumento do volume com aumentos significativos na frequência respiratória.

Em relação a VEF1 (Tabela 4), que é uma variável que indica o volume de ar que é exalado no primeiro segundo durante a manobra de CVF, indicando a quantidade de ar eliminada no primeiro segundo da manobra expiratória forçada, sendo, portanto, a medida de função pulmonar mais útil clinicamente, não foram observadas melhorias significativas nesta variável entre as mulheres após a cirurgia, em nenhum dos grupos, considerando a faixa etária e o grau de obesidade. 
Tabela 4 - Distribuição da referência teórica e das estimativas da variável espirométrica VEF1. Rio de Janeiro, RJ, Brasil, 2018

\begin{tabular}{ccccc} 
Paciente & Teórica & Pré-Cir. & 1 $^{\text {a }}$ Pós-Cir. & 2 $^{\text {a }}$ Pós-Cir. \\
\hline 1 & 2,34 & 2,86 & 2,65 & 2,74 \\
\hline 2 & 3,45 & 2,75 & 3,16 & 2,76 \\
\hline 3 & 2,96 & 2,99 & 2,94 & 2,85 \\
\hline 4 & 2,07 & 1,97 & 2,17 & 2,26 \\
\hline 5 & 2,77 & 2,15 & 2,06 & 2,06 \\
\hline 6 & 2,91 & 3,33 & 3,29 & 3,26 \\
\hline 7 & 2,74 & 2,48 & 2,35 & 2,43 \\
\hline 8 & 2,7 & 2,2 & 2,56 & 2,26 \\
\hline 9 & 2,29 & 2,52 & 2,55 & 2,48 \\
\hline 10 & 2,51 & 2,6 & 2,77 & 2,65 \\
\hline 11 & 2,37 & 2,35 & 2,2 & 2,14 \\
\hline 12 & 2,46 & 1,69 & 1,74 & 1,8 \\
\hline 13 & 2,45 & 1,8 & 1,75 & 1,56 \\
\hline 14 & 2,51 & 1,96 & 1,88 & 1,89 \\
\hline Média & 2,6 & 2,4 & 2,43 & 2,36 \\
\hline DP & 0,34 & 0,47 & 0,49 & 0,46
\end{tabular}

Foram calculados o OR, considerando a medida da VEF1 antes da cirurgia e 60 dias depois, entre os grupos de mulheres com obesidade grau l, comparado a mulheres com obesidade grau II e entre o grupos de mulheres com obesidade grau II comparado com - grupo de mulheres grau III, para avaliar a razão de chance de melhora da VEF1 após a realização da cirurgia nesses grupos. A mesma comparação foi feita em relação à faixa etária, comparando mulheres entre 35 e 45 anos, com as mulheres com mais de 45 anos.

O OR na primeira comparação (obesidade grau I x obesidade grau II) foi de 0,66 (IC $95 \%: 0,03$ a 11,28$) p=0,77$. Na segunda comparação (obesidade grau II $x$ obesidade grau III), o OR foi de 0,50 (IC $95 \%$ : 0,03 a 6,68) p=0,60 e na terceira comparação (obesidade grau I x obesidade grau III), o OR foi de 0,66 (IC 95\%: 0,03 a 11,28) $p=0,77$. Em relação à faixa etária e o VEF1, o OR foi de 0,75 (IC 95\%: 0,07 a 7,21) $p=0,80$. Em nenhuma das comparações foi observado significância estatística.

O diagnóstico de distúrbio obstrutivo é obtido a partir da razão entre as duas medidas. O resultado depende de equação que é determinada conforme o paciente. Não foram observadas mudanças nessa variável após a realização da cirurgia.

Como podemos observar na Tabela 5, com exceção das obesas mórbidas (pacientes 10,11 e 13), todas as outras pacientes apresentaram VÉF1/CVF na fase pré-cirurgia dentro dos valores teóricos esperados. Na maioria das pacientes observou-se uma redução da VEF1/CVF, determinando uma redução média de 83,58 L, para 82,30L $(-1,28 \mathrm{~L})$. 
Tabela 5 - Distribuição da referência teórica e das estimativas da variável espirométrica VEF1/CVF. Rio de Janeiro, RJ, Brasil, 2018

\begin{tabular}{ccccc} 
Paciente & Teórico & Pré-Cir. & 1 $^{\text {a }}$ Pós-Cir. & 2a Pós-Cir. \\
\hline 1 & 83,11 & 88,22 & 80,93 & 81,45 \\
\hline 2 & 84,85 & 85,83 & 85 & 84,89 \\
\hline 3 & 82,6 & 86,54 & 86,15 & 85,19 \\
\hline 4 & 81,96 & 81,79 & 86,03 & 85,77 \\
\hline 5 & 80,18 & 81,04 & 78 & 76,29 \\
\hline 6 & 85,23 & 87,04 & 87,45 & 88,17 \\
\hline 7 & 85,03 & 81,25 & 79,95 & 81,46 \\
\hline 8 & 81,08 & 81,36 & 80,07 & 78,93 \\
\hline 9 & 87,81 & 80,89 & 80,37 & 80,3 \\
\hline 10 & 84,82 & 81,34 & 82,03 & 83,15 \\
\hline 11 & 85 & 84,39 & 83,21 & 88,59 \\
\hline 12 & 83,55 & 78,37 & 82,33 & 77,03 \\
\hline 13 & 83,69 & 73,72 & 72,42 & 78,26 \\
\hline 14 & 81,25 & 82,28 & 83,59 & 82,83 \\
\hline Média & 83,58 & 82,43 & 81,96 & 82,3 \\
\hline DP & 2,05 & 3,8 & 3,88 & 3,91
\end{tabular}

Mulheres com idade inferior a 45 anos apresentaram 1,2 vezes mais chances de aumentar a relação VEF1/CVF após a cirurgia do que aquelas com mais de 45 anos (OR de 1,2 IC 95 \%: 0,13 a 11,05), porém, sem apresentar significância estatística $p=0,87$.

Apenas entre as obesas com grau I, a VEF1/CVF apresentou melhora em relação ao valor teórico esperado $(81,25 \mathrm{~L})$ e após a realização da cirurgia. Na primeira medida, em até 30 e 60 dias após a realização da cirurgia, a VEF1/CVF foi respectivamente de 83,59 L e $82,83 \mathrm{~L}$, com incremento de 1,31 e 0,55 L, respectivamente.

A chance de mulheres com obesidade grau I melhorarem a relação VEF1/CVF quando comparadas às mulheres com obesidade grau II após a realização da cirurgia, foi 1,2 maior, porém, sem significância estatística (OR 1,2 IC 95\%: 0,13 a 11,05) $p=0,87$.

Comparando as mulheres com obesidade grau II, com as mulheres com obesidade grau III, O OR foi de 0,33 (IC 95\%: 0,02 a 5,32), também sem significância estatística $(\mathrm{p}=0,43)$. Quando comparamos as mulheres com obesidade grau I com mulheres com obesidade grau III, O OR foi de 0,08 (IC 95 \%: 0,003 a 1,94), também sem significância estatística $(p=0,12)$. Todas as comparações consideram a primeira e a terceira medidas espirométricas (antes da cirurgia e 60 dias depois).

O Fluxo expiratório forçado médio (FEFx-y\%) representa o fluxo expiratório forçado médio de um segmento obtido durante a manobra de CVF. O FEF25-75\% é o fluxo expiratório forçado médio na faixa intermediária da CVF, isto é, entre 25 e $75 \%$ da curva de CVF O fluxo expiratório forçado médio (FEF 25-75\%), indicando um parâmetro obtido durante a manobra de CVF.

A exemplo do que foi observado em relação às variáveis anteriormente apresentadas, a realização da cirurgia teve um impacto muito discreto nessa variável também. Entretanto, 
seis pacientes (50\%) apresentaram discreto aumento na FEF $25-75 \%$ quando comparado ao valor teórico esperado. Mesmo entre as obesas mórbidas, a melhora na relação FEF $25-75 \%$ foi constatada. Das três (25\%) pacientes nessa condição de obesidade, somente em uma não foi observada a melhora, justamente na paciente com mais idade (48 anos).

O OR de mulheres com idade inferior a 45 anos aumentar a FEF $25 \%-75 \%$ após a cirurgia quando comparado que aquelas com mais de 45 anos foi de 0,08 (IC $95 \% \mathrm{Cl}$ : 0,003 a 1,94) porém, sem apresentar significância estatística $(p=0,12)$.

O OR entre mulheres com obesidade grau I quando comparadas às mulheres com obesidade grau II após a realização da cirurgia, em relação à melhora da FEF 25\%-75\% foi de 0,20 (IC $95 \%$ : 0,01 a 3,66) p =0,27, sem significância estatística.

Comparando as mulheres com obesidade grau II, com as mulheres com obesidade grau III, O OR foi de 0,60 (IC $95 \%$ : 0,026 a 13,58) $p=0,74830$. Quando comparamos as mulheres com obesidade grau I com mulheres com obesidade grau III, o OR foi de 3,0 (IC 95\%: 0,15 a 59,89, também sem significância estatística ( $p=0,47)$. Todas as comparações consideram a primeira e a terceira medidas espirométricas (antes da cirurgia e 60 dias depois).

No que se refere à qualidade de vida, todas as mulheres disseram que houve mudança significativa em sua vida após a realização da cirurgia. Elas destacaram que houve melhora na sua autoestima, sentem-se mais bonitas, mais bem-dispostas e referem menos dores na coluna. Passaram a ter um estado físico mais compatível com suas idades e estão muito satisfeitas com o resultado da cirurgia.

\section{DISCUSSÃO}

A gigantomastia é uma condição que afeta mulheres jovens e adultas em todas as faixas etárias, trazendo uma série de prejuízos e impactos físicos e psicossociais negativos, sendo as doenças musculoesqueléticas e a dor crônica como resultados do aumento das mamas $^{(1)}$.

Em alguns casos de gigantomastia, a mama pode crescer $13-23 \mathrm{~kg}$, levando a uma significativa carga física e mental, o que pode diminuir drasticamente a qualidade de vida dessas mulheres ${ }^{(1-2)}$.

Nos tratados cirurgicamente nesse estudo, todas as 14 mulheres (100\%) apresentavam gigantomastia, e algum tipo de sofrimento físico e psicossocial. A indicação cirúrgica teve como objetivo ressecar o excesso de tecido mamário para proporcionar alívio nos sintomas físicos, principalmente aqueles relacionados à respiração.

No entanto, os resultados demonstraram que o tratamento cirúrgico da gigantomastia não foi tão eficaz para a melhoria dos resultados da espirometria, em que pese o fato de termos constatado, na análise qualitativa, relatos de diminuição das reclamações de dor relacionada ao tamanho da mama. Talvez, com maior tempo de seguimento após a realização da cirurgia, pudéssemos ter encontrado melhores resultados na espirometria ${ }^{(4)}$.

A própria condição pós-operatória, considerando o tempo entre a realização da espirometria pós-cirurgia (30 dias e em até 60 dias) pode ter sido um fator de confusão que comprometeu a realização do exame, e impactou pouco na melhoria da função respiratória pós-gigantoplastia, em relação às variáveis estudadas ${ }^{(5)}$.

Cabe destacar que somente duas (13,33\%) das mulheres nesse estudo apresentavam valores de CVF, teste de função pulmonar mais importante a ser considerado nos casos de gigantomastia, acima do valor esperado, que demonstra o quanto a condição dessas mulheres, todas obesas, impactam na função respiratória. 
Com relação à VVM, o discreto incremento de 1,65 L/min, após 60 dias da cirurgia não foi significativamente estatístico, a exemplo do que foi encontrado em outras variáveis analisadas na espirometria. Mulheres com obesidade grau I parecem ser aquelas que mais podem se beneficiar em relação VEF1/CVF com a realização da cirurgia de gigantoplastia. Quando comparadas as mulheres com obesidade grau II, a chance de melhorar essa relação foi 1,2 maior, porém, sem significância estatística (OR 1,2 IC 95\%: 0,13 a 11,05) $p=0,87$.

As mulheres com idade inferior a 45 anos parecem ter mais chances de serem beneficiadas com a realização da cirurgia no que se refere à melhora da relação VEF1/CVF, quando comparadas as mulheres com mais idade (OR de 1,2 IC $95 \%$ : 0,13 a 11,05), porém, sem apresentar significância estatística $(p=0,87)$.

Embora o IMC possa estar associado com complicações pós-operatórias(7), não foi observado na amostra qualquer tipo de complicações que pudesse estar associada ao IMC, o que faz acreditar que a cirurgia de gigantoplastia é uma intervenção que, mesmo em mulheres com sobrepeso e obesidade, pode ser vantajosa.

O tempo de seguimento e os intervalos entre a realização das espirometrias póscirurgia devem ser considerados como limitações do estudo que podem impactar nos resultados encontrados. O ideal seria um seguimento de no mínimo 6 meses a 5 anos, o que infelizmente não foi possível por conta do prazo exíguo para a conclusão do estudo, desenvolvido por ocasião de um estágio de pós-doutoramento.

\section{CONCLUSÃO}

Mamoplastia de redução em mulheres portadoras de gigantomastia pode melhorar a qualidade de vida dessas mulheres, com redução da intensidade de sintomas como a dor na coluna e a indisposição para a realização de tarefas do cotidiano, conforme autorrelato das mulheres que participaram do estudo. Não observamos significância que permitisse associar a mamoplastia com a melhora da função respiratória.

Importante destacar ainda que a gigantomastia é um problema que esteve associado nesse estudo a impactos negativos, independentemente de serem mulheres obesas ou não e independente do grau de obesidade, sobretudo no que se refere à qualidade de vida e função respiratória.

\section{REFERÊNCIAS}

1. Chetty V, Ndobe E. Macromastia and gigantomastia: efficacy of the superomedial pedicle pattern for breast reduction surgery. S. Afr. j. surg. [Internet]. 2016 [acesso em 20 dez 2018]; 54(4): 46-50. Disponível em: http://www.scielo.org.za/scielo.php?script=sci arttext\&pid=S0038-23612016000400011\&lng=en.

2. Andrade AC, Veiga DF, Aguiar IC, Juliano Y, Sabino-Neto M, Ferreira LM. Outcomes analysis of breast reduction in Brazilian women using the BREAST-Q® questionnaire: a cross-sectional controlled study. Clinics. [Internet]. 2018 [acesso em 20 dez 2018]; 73. Disponível em: http://dx.doi.org/10.6061/ clinics/2018/e313.

3. Cunha MS, Santos LL, Viana AA, Bandeira NG, Lima FJA, Meneses JVL. Avaliação da função pulmonar em pacientes submetidas à mastoplastia redutora. Rev. Col. Bras. Cir. [Internet]. 2011 [acesso em 20 dez 2018]; 38(1): 11-14. Disponível em: http://dx.doi.org/10.1590/S0100-69912011000100003.

4. Machado JRS, Bilheri DFD, Tomasi LL, Steidl EMS, Mancopes R. Respiratory muscle training outcomes on swallowing biomechanics and respiratory function measures in normal subjects. Rev. CEFAC.

[Internet]. 2018 [acesso em 20 dez 2018]; 20(6): 778-784. Disponível em: http://dx.doi.org/10.1590/1982021620182069417. 
5. Gutiérrez CM, Beroiza WT, Borzone TG, Caviedes SI, Céspedes GJ, Gutiérrez NM, et al. Espirometría: manual de procedimientos. Rev. chil. enferm. respir. [Internet]. 2018. [acesso em 20 dez 2018]; 34(3): 171188. Disponível em: http://dx.doi.org/10.4067/s0717-73482018000300171.

6. Rocha MRS da, Souza S, Costa CM da, Merino DFB, Montebelo MIL, Rasera-Júnior I, et al. Airway positive pressure vs. exercises with inspiratory loading focused on pulmonary and respiratory muscular functions in the postoperative period of bariatric surgery. $A B C D$, arq. bras. cir. dig. [Internet]. 2018. [acesso em 20 dez 2018]; 31(2). Disponível em: http://dx.doi.org/10.1590/0102-672020180001e1363.

7. Parikh M, Chung M, Sheth S, McMacken M, Zahra T, Saunders JK, et al. Randomized pilot trial of bariatric surgery versus intensive medical weight management on diabetes remission in type 2 diabetic patients who do NOT meet NIH criteria for surgery and the role of soluble RAGE as a novel biomarker of success. Ann Surg. [Internet]. 2014[acesso em 20 dez 2018]; 260(4):617-22. Disponível em: https://www. ncbi.n/m.nih.gov/pubmed/25203878.

Recebido: 20/12/2018

Finalizado: 11/07/2019

Autor Correspondente:

Carlos Roberto Lyra da Silva

Universidade Federal do Estado do Rio de Janeiro

Av. Pasteur, 296 - 22290-240 - Rio de Janeiro, RJ, Brasil

E-mail: profunirio@gmail.com

Contribuição dos autores:

Contribuições substanciais para a concepção ou desenho do estudo; ou a aquisição, análise ou interpretação de dados do estudo - OJS, RCLS

Elaboração e revisão crítica do conteúdo intelectual do estudo - OJS, CLS, TQL, DAM, KGNSA

Aprovação da versão final do estudo a ser publicado - CLS, RCLS 